\title{
Hardwired Configurable Photonic Integrated Circuits Enabled by 3D Nanoprinting
}

\author{
T. Hoose(1,2), M. Blaicher(1,2), J. N. Kemal(2), H. Zwickel(2), M. R. Billah(1,2), P.-I. Dietrich ${ }^{(1,2,3)}$, \\ A. Hofmann (4), W. Freude(2), S. Randel(2), C. Koos ${ }^{(1,2,3)}$ \\ (1) Institute of Microstructure Technology (IMT), Karlsruhe Institute of Technology (KIT), 76344 Eggenstein- \\ Leopoldshafen, Germany, tobias.hoose@kit.edu, christian.koos@kit.edu \\ (2) Institute of Photonics and Quantum Electronics (IPQ), KIT, 76131 Karlsruhe, Germany \\ (3) Vanguard Photonics GmbH, 76344 Eggenstein-Leopoldshafen, Germany \\ (4) Institute for Automation and Applied Informatics, KIT, 76344 Eggenstein-Leopoldshafen, Germany
}

Abstract We propose and experimentally demonstrate a novel class of photonic integrated circuits (PIC) that can be configured by hardwiring of functional building blocks using 3D-printed single-mode waveguides. The concept allows realizing a multitude of functionalities with a single PIC design.

\section{Introduction}

Photonic integration has experienced tremendous progress over the last decade, and several platforms have reached industrial maturity. The most prominent example is silicon photonics (SiP), which exploits highly developed CMOS processes for low-cost mass-production of photonic integrated circuits (PIC). Design of these PIC can rely on advanced device libraries that offer a comprehensive portfolio of standardized building blocks with proven performance, which allow to address a wide variety of applications with small technological overhead. For these circuits, design and mask fabrication represent the main cost drivers, whereas largescale mass fabrication of a specific PIC design is available at rather low cost through fabrication facilities worldwide. However, this situation is somewhat in contrast to the fact that photonics still represents a highly fragmented market covering a wide variety of applications that require low or medium quantities of applicationspecific PIC rather than large quantities of the same PIC. As a consequence, highly scalable photonic integration can currently not unfold its full economic strength.

This contradiction could be overcome by circuit architectures that contain a multitude of generic building blocks that can be connected to provide specific functionalities in a separate configuration step. Configurable PIC have been previously demonstrated, exploiting, e.g., meshes of thermo-optic phase shifters and power splitters that can be programmed by setting the various phase shifts ${ }^{1}$. However, the functionality of such mesh-based photonic signal processors is essentially restricted to different kinds of passive optical filters, and it crucially relies on the precise setting and permanent control of a large number of electrical signals.

In this paper we introduce and experimentally demonstrate the concept of hardwired configurable PIC (HC-PIC) that allows to flexibly combine a multitude of standard building blocks for, e.g., light manipulation, routing, and detection. The application-specific functionality of HC-PIC is established by the concatenation of functional blocks in a one-time configuration step. The generic PIC relies on high-throughput production of standardized designs in advanced large-scale fabrication facilities. The application-specific circuit configuration is accomplished during the packaging process by connecting suitable ports of an optical wire board using 3D direct-write laser lithography. By an appropriate choice of onchip building blocks, the same PIC arrangement can be used to serve a wide variety of applications. In our experiments, we demonstrate the viability of the HC-PIC concept by using a circuit design that can be configured as a transmitter for conventional on-off-keying (OOK) signals, or as a self-homodyne transmitter. To the best of our knowledge, this is the first demonstration of a hardwire-configurability in photonic integration.

\section{Hardwired configurable PIC (HC-PIC)}

The schematic concept of an HC-PIC is illustrated in Fig.1. The circuit relies on a SiP chip, which consists of a variety of building blocks such as coupling elements for off-chip connections to external fibers and light sources, phase shifters and electro-optic modulators for light manipulation, passive elements such as power splitters and combiners for routing the light, and also photodetectors. All building blocks are connected to a central optical wire board (OWB), which allows to configure the functionality of the PIC by establishing appropriate connections between the various optical ports. Free configurability of the connections requires in-situ fabrication of compact waveguides that allow low-loss and low crosstalk single-mode transmission. This is achieved by using high-resolution two-photon lithography to create $3 D$ freeform waveguide structures. After lithography and development, the free-standing waveguides are embedded into a low refractive-index cladding that acts as a protective cover and as a low-index cladding material. Note that wiring of the OWB relies on the same tools and processes as the concept of photonic wire bonds ${ }^{2}$ (PWB), which can be used for connecting HC-PIC to optical fibers ${ }^{3}$ and external light sources ${ }^{4}$, or for realizing crossingfree $\mathrm{PIC}^{5}$. Packaging and configuration of the 

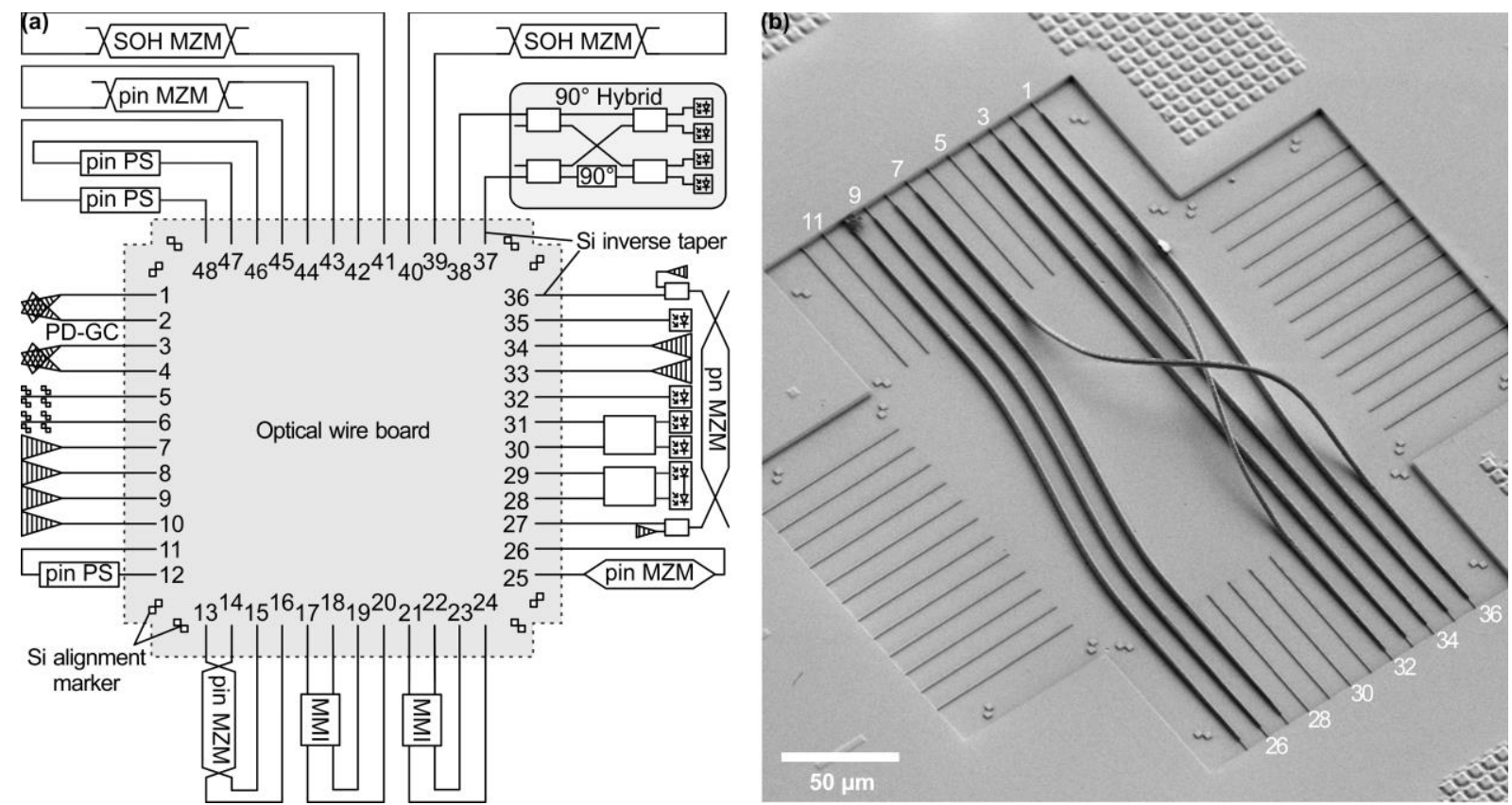

Fig. 1: Schematic of the HC-PIC concept. (a) The demonstration relies on a generic silicon PIC which comprises a variety of coupling elements for off-chip connections to optical fibers and external light sources, a number of phase shifters and electrooptic modulators for light manipulation, passive elements such as power splitters and combiners, and finally photodetectors for light detection. All these building blocks are connected to a central optical wire board (OWB), which allows to freely configure the functionality of the PIC by establishing appropriate connections between the various optical ports $1 \ldots 48$. (SOH = siliconorganic hybrid ${ }^{6}, \mathrm{pn} \mathrm{MZM}=$ pn depletion-type Mach-Zehnder modulator, pin MZM = pin Mach-Zehnder modulator, $\mathrm{PS}=$ phase shifter, $\mathrm{MMI}=$ multimode interference coupler, $\mathrm{PD}-\mathrm{GC}=$ polarization-diversity grating coupler $^{7}$ ) (b) Scanning electron microscope (SEM) image of a configured OWB: Free configurability of the connections relies on in-situ fabrication of 3D freeform single-mode waveguides between the chosen optical ports. The free-standing waveguides are embedded in a low refractiveindex cladding (not shown). Wiring of the OWB relies on the same tools and processes as photonic wire bonding (PWB $)^{2-5}$. Packaging and configuration of the FC-PIC can hence be combined in a single fabrication step.

HC-PIC can hence be combined in a single fabrication step.

\section{HC-PIC demonstration}

For demonstrating the viability of the HC-PIC, the generic PIC shown in Fig. 1(a) was fabricated using the foundry service by the $A^{*}$ Star Institute of Microelectronis (IME) in Singapore. Fig. 1(b) shows a scanning electron microscope (SEM) image of the OWB with 48 ports that can be freely connected by 3D-printed single-mode waveguides having an rectangular cross-section of $2.0 \mu \mathrm{m} \times 1.8 \mu \mathrm{m}$. They were fabricated using a commercially available photoresist (IP-Dip by Nanoscribe $\mathrm{GmbH}$ ) with a refractive index of $n_{\text {core }}=1.53$. This leads to single-mode operation when embedding the OWB connections into a low refractive index cladding of $n_{\text {clad }} \approx 1.36$. The OWB configuration shown in Fig. 1(b) was chosen for evaluating the insertion loss (IL) of the optical connections and to demonstrate the viability of the HC-PIC approach. The IL of the various connections was measured by comparing the transmission through the OWB connections to the transmission of an equivalent arrangement, but without an OWB connection. The results of the IL measurements are given in Table 1. Note that the losses are significantly higher for OWB connections $(7-36)$ and $(2-32)$ with longer non-planar crossing trajectories as compared to a shorter planar waveguide $(4-33)$. We expect that these losses can be significantly reduced by an improved design of the OWB (e. g., in circular arrangement). The IL of OWB connections (9$26)$ and $(25-10)$ cannot be separated, since both connections are part of a common optical path. We can hence only specify the overall IL of both structures, which amounts to approximately $1 \mathrm{~dB}$.

\section{Optical transmitters with a HC-PIC}

We use our PIC design Fig. 1(a) to realize two different optical transmitters that we test in communication experiments. The first transmitter (Tx) consists of two grating couplers used as input and output ports which are connected to a pn-type MZM via OWB connections, marked in red in Fig. 2 (a). For the transmission experiment, the MZM is driven by an arbitrary-waveform generator (AWG) through drive amplifiers and microwave probes in ground-signal-signal-ground (G-S-S-G) configuration. An erbium doped fiber amplifier (EDFA) is used to compensate fiberchip coupling and on-chip losses of the device, which total to $18 \mathrm{~dB}$. The IL of the concatenated OWB connections,

(7) $-(36)$ and (27) - (34), amounts up to $4.0 \mathrm{~dB}$. Also in this configuration, we cannot separate the individual IL of the two OWB connections. The EDFA is
Table 1: Measured insertion loss (IL) of OWB connections

\begin{tabular}{c|c} 
OWB connection & $\mathrm{IL}[\mathrm{dB}]$ \\
\hline $1-35$ & 1.2 \\
\hline $2-32$ & 2.9 \\
\hline $3-34$ & 1.0 \\
\hline $4-33$ & 0.5 \\
\hline $7-36$ & 4.8 \\
\hline $8-27$ & 0.47 \\
\hline$(9-26)+(25-10)$ & 1.0
\end{tabular}




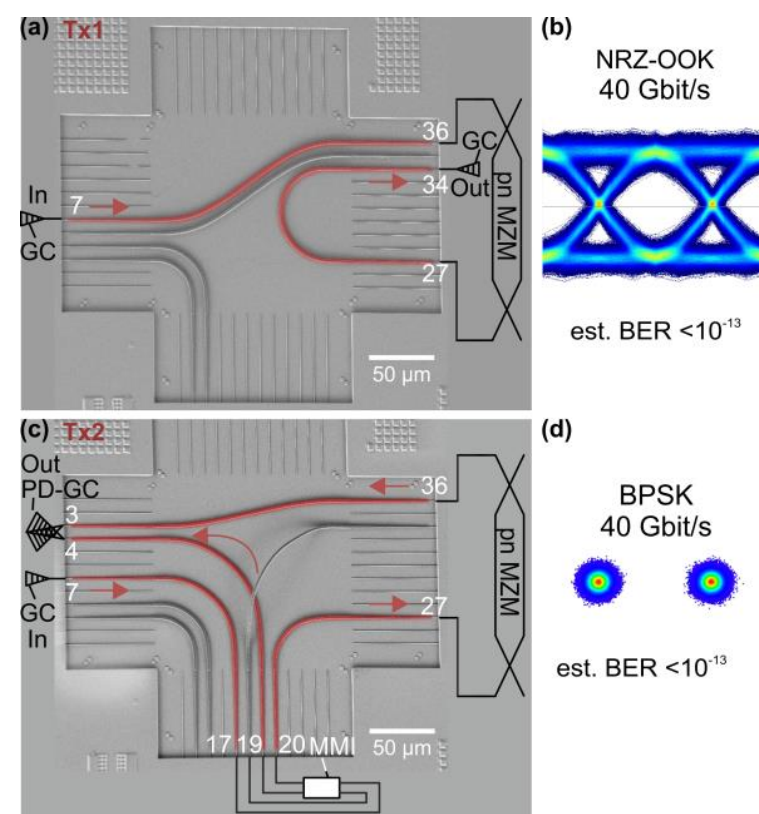

Fig. 2: Optical transmitters on a HC-PIC. Both transmitters operate with an external light source. Functionally relevant OWB connections are marked in red. The signal propagation direction is given by arrows. (a) Single-channel optical transmitter Tx1 for conventional on-off-keying (OOK) data signals. The input ("In", Port 7) and output ("Out", Port 34) ports are on opposite sides of the die for an easy coupling with standard single-mode fibers (SMF). (b) Recorded eye diagram generated by Tx1 in (a). We transmit NRZ-OOK signals with data rates up to $40 \mathrm{Gbit} / \mathrm{s}$. The BER was estimated via a measured Q-factor. (c) Self-homodyne transmitter Tx2. Light is coupled to the PIC via a single GC at Port 7 and is then guided through the OWB connection ( 7 17 ) to a multimode interference coupler (MMI). Part of the light is routed to the pn MZM at Port 27 and then coupled to a linear polarization state of the outgoing SMF by a polarizationdiversity grating coupler (PD-GC). Another part of the light is left unmodulated and routed straight to the PD-GC, which couples to the orthogonal linear polarization state of the SMF. The modulated and the orthogonally polarized unmodulated carrier are co-propagated in the SMF to a remote homodyne receiver. (d) Measured constellation diagram for binary phase shift keying (BPSK) at $40 \mathrm{Gbit} / \mathrm{s}$. The BER was estimated via a measured EVM.

followed by a bandpass filter to suppress out-ofband amplified spontaneous emission (ASE) noise in front of a photoreceiver (not shown). The optical signal is recorded with a high-speed oscilloscope for offline processing. An eye diagram of a NRZ-OOK signal at a data rate of $40 \mathrm{Gbit} / \mathrm{s}$ is shown in Fig. 2(b). From the measured $\mathrm{Q}$-factor we estimate a bit error ratio (BER) below $10^{-13}$. The transmitter Tx2 with copropagation local oscillator is demonstrated in a similar experiment, see Fig. 2(c). The device consists of a GC for light input (Port 7), a multimode-interference coupler (MMI) that acts as a power splitter, a pn-type MZM at Port 27, and a $\mathrm{PD}-\mathrm{GC}^{7}$ for coupling the light to a transmitting SMF in a linear state of polarization (SOP). The unmodulated local oscillator from Port 19 is coupled to an orthogonal SOP in the SMF and co-propagates with the modulated signal. All components are connected via four OWB connections. The transmitted signals in the SMF are then amplified by an EDFA, and sent through a bandpass filter for ASE noise suppression. The signal is detected by a self-homodyne receiver built from discrete components, and recorded by an optical modulation analyzer (OMA, Keysight N4391). The measured constellation diagram for binary phase shift keying (BPSK) at $40 \mathrm{Gbit} / \mathrm{s}$ is shown in Fig 2(d). The BER estimated from the error-vector magnitude signal is below $10^{-13}$.

\section{Summary}

We introduced and experimentally demonstrated the concept of a hardwired configurable photonic integrated circuit (HC-PIC), which exploits in-situ 3D nanoprinting of single-mode connections, which link optical blocks with different functionality via an optical wire board (OWB). We show the viability of the concept with a generic silicon PIC. We realize two different kinds of optical transmitters - Tx1 for standard on-off-keying (OOK) signals, and Tx2 for BPSK data transmission and reception with a self-homodyne scheme. The HCPIC concept can be extended to other photonic integration platforms, and might even permit to combine different material systems with complementary functionalities in a hybrid approach. To the best of our knowledge, this is the first demonstration of a hardwired configurable photonic integration. We believe that the HC-PIC concept will allow to exploit the unprecedented scalability advantages of high-density photonic integration for realizing application-specific PIC by hardwiring an appropriate configuration of optical building blocks.

Acknowledgements: This work was supported by the joint BMBF Project PHOIBOS (grant 13N12574) and PRIMA (grant 13N14630), by the Deutsche Forschungsgemeinschaft (DFG) through the CRC WavePhenomena (\# 1173), the Helmholtz International Research School for Teratronics (HIRST), the European Research Council (ERC Starting Grant 'EnTeraPIC', \# 280145; ERC Consolidator Grant 'TeraSHAPE', \# 773248), the H2020 Photonic Packaging Pilot Line PIXAPP (grant 731954) and the Alfried Krupp von Bohlen und Halbach Foundation. Our chips were fabricated using the OpSIS service, through IME A*STAR in Singapore.

\section{References}

[1] D. Pérez et al., "Multipurpose Silicon Photonics Signal Processor Core," Nat. Comm., vol. 8, Article number: 636 (2017).

[2] N. Lindenmann et al., "Photonic Wire Bonding: A Novel Concept for Chip-Scale Interconnects," Opt. Express, Vol. 20, no. 16 , p. 17667 (2012)

[3] N. Lindenmann et al., "Connecting Silicon Photonic Circuits to Multi-Core Fibers by Photonic Wire Bonding," J. Lightwave Technol., Vol. 33, no. 4, p. 755 (2015).

[4] M. R. Billah et al., "Hybrid Integration of Silicon Photonics Circuits and InP Lasers by Photonic Wire Bonding," arXiv:1802.03454 (2018)

[5] A. Nesic et al., "Hybrid 2D/3D Photonic Integration for Non-Planar Circuit Topologies," 42th European Conf. Opt. Commun., Düsseldorf, Germany, Sept. 18-22 (2016)

[6] C. Koos et al., "Silicon-Organic Hybrid (SOH) and Plasmonic-Organic Hybrid (POH) Integration," J. Lightw.Technol. 34, 256-268 (2016).

[7] F. Van Laere et al., "Focusing Polarization Diversity Grating Couplers in Silicon-on-Insulator," J. Lightw. Technol. 27, 612-618 (2009). 